\title{
MiR-371 promotes proliferation and metastasis in hepatocellular carcinoma by targeting PTEN
}

\author{
Hao Wang ${ }^{1, \#}$, Yi Zhao ${ }^{1, \#}$, Tingsong Chen ${ }^{2}$, Guofang Liu ${ }^{1}, \mathrm{Nan} \mathrm{He}^{3} \mathcal{E}$ Heping $\mathrm{Hu}^{1, *}$ \\ ${ }^{1}$ Eastern Hepatobiliary Surgery Hospital (EHBH), Second Military Medical University, Shanghai City 200438, ${ }^{2}$ The Seventh People's \\ Hospital of Shanghai, Shanghai City 200137, ${ }^{3}$ Guangdong Ascendas Genomics Technology Co., Ltd., Zhongshan 519000, China
}

\begin{abstract}
Hepatocellular carcinoma (HCC) is the leading cause of cancer-related mortality worldwide. MiR-371 has recently emerged as an important regulator in tumorigenesis, and may serve as a biomarker for malignant tumors. We transfected miR-371 or its inhibitor in two human HCC cell lines, then used 3-(4,5-Dimethylthiazol-2-yl)-2,5-diphenyltetrazolium bromide, soft agar colony formation, and transwell migration assays to evaluate the effects on cell proliferation, migration, and invasion. We found that miR-371 was positively correlated with HCC metastasis and poor prognosis in the inflicted patients, and the high expression of miR-371 was promoted, whereas a low level of miR-371 depressed cell proliferation and invasion. We found PTEN to be a direct target of miR-371. The overexpression or knockdown of PTEN exhibited the opposite effects from those of miR-371 on cell proliferation and migration. Our study demonstrates that miR-371 promotes proliferation and metastasis in HCC by targeting PTEN. [BMB Reports 2019; 52(5): 312-317]
\end{abstract}

\section{INTRODUCTION}

Most cases of hepatocellular carcinoma (HCC) occur in the Asia-Pacific region, where chronic hepatitis B infection is endemic. The other main risk factors of HCC include infection with hepatitis $\mathrm{C}$ virus $(\mathrm{HCV})$ and cirrhosis (1). Despite the great progress that has been achieved in current therapeutics, ineffective medical intervention, relapse, and metastasis are still frequently observed in clinical settings, indicating that more intimate understanding of the complex regulatory mechanism of HCC is needed, along with novel therapeutics.

*Corresponding author. Tel: +86-136-0173-0528; Fax: +86-1360173-0528; E-mail: huehbh@sina.com

${ }^{\#}$ These authors contributed equally to this work.

https://doi.org/10.5483/BMBRep.2019.52.5.155

Received 19 July 2018, Revised 9 August 2018,

Accepted 19 September 2018

Keywords: Cancer, Hepatocellular carcinoma, Metastasis, miR-371, PTEN
Accumulating evidence suggests that microRNAs (miRNAs, miRs) play essential roles in regulating the development, regeneration, and metabolism of the liver. The dysregulation of miRNA has been associated with liver diseases including cirrhosis, steatosis, hepatitis, and HCC. An exceptional example is miR-122, which is the most abundant miRNA in the adult liver and which plays a central role in liver biology and disease. It has been shown that miR-122 is an essential host factor for the infection of HCV, and thus an anti-viral target. MiRNA-based therapeutics have shown promise for treating liver disease, including viral hepatitis (2).

Meanwhile, miRNA pairing with mRNA is essentially based on only seven nucleotides (miRNA seed) in the miRNA, so there is a relatively high probability that the sequence may exist in many mRNA molecules, meaning that a single miRNA may target many different mRNAs. The multi-target feature brings about the prospect of miRNA serving as a potent "magic bullet" for complex diseases such as cancer (3). Thus, identifying miRNA with specific function constitutes an important direction. Recent studies have implicated specific miRNAs in cellular transformation and tumorigenesis, and have highlighted the role of tumor-associated miRNAs in cancer. Specifically, more and more miRNAs are reported to be involved in HCC progression, and the potential of several tumor suppressor miRNAs in cancer gene therapy has been confirmed (4).

Emerging evidence suggests that a novel miRNA miR-371 may play important roles in tumorigenesis. For example, miR-371 promotes cell proliferation in pancreatic cancer and reduces patient survival (1), and is also upregulated in esophageal squamous cell carcinoma (5). Serum miR-371 may serve as a biomarker for malignant germ cell tumors (GCTs) (6) and testicular germ cell tumors (7). These points of evidence suggest that miR-371 may function generally in cancer, which prompted us to study its involvement in human HCC.

\section{RESULTS}

MiR-371 is correlated with poor prognosis in HCC patients In order to explore the function of miRNAs in HCC, we conducted a correlational analysis on the relationship between miRNA expression level and metastasis (Fig. 1A) and survival (Fig. 1B) based on the normalized Gene Expression Omnibus

ISSN: 1976-670X (electronic edition)

Copyright (C) 2019 by the The Korean Society for Biochemistry and Molecular Biology

c) This is an open-access article distributed under the terms of the Creative Commons Attribution Non-Commercial License (http://creativecommons.org/licenses/by-nc/4.0) which permits unrestricted non-commercial use, distribution, and reproduction in any medium, provided the original work is properly cited. 
A
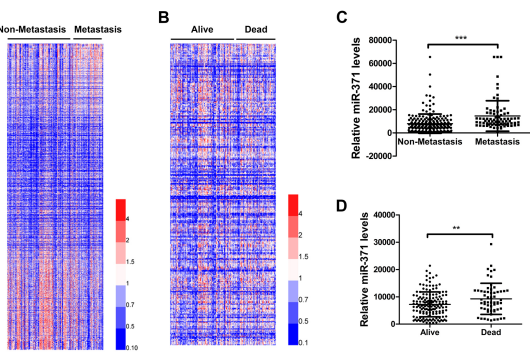

Fig. 1. miR-371 is correlated with poor prognosis in HCC patients. (A) Heat map represents the expression levels of microRNAs in patients with or without metastasis in the GSE6857 HCC dataset. (B) Heat map represents the expression levels of microRNAs in patients with different survival outcomes in GSE6857 HCC dataset. (C) The miR-371 level in metastasis or metastasis-free HCC patients in GSE6857 datasets. (D) The miR-371 level in different survival outcomes of HCC patients in GSE6857 datasets. Data are shown as mean \pm S.D. ${ }^{* P}<0.05$; $* * \mathrm{P}<0.01 ; * * * \mathrm{P}<0.001 ; \mathrm{ns}$, not significant (Student's t-test).

(GEO) dataset (GSE6857) with published gene expression data and survival information (8). In the GSE6857 data, we found that miR-371 tended to show a higher expression level in patients with metastasis (Fig. 1C) as well as a shorter survival time (Fig. 1D), suggesting that miR-371 is likely an indicator of poor prognosis of human HCC, which prompted us to focus on miR-317 in this study.

\section{MiR-371 promotes HCC cells proliferation}

We next explored the function of miR-371 in two lines of human hepatoma cells: Hep3B and HepG2. We analyzed the impact of miR-371 on human hepatoma cell proliferation by monitoring cell viability for five consecutive days using 3-(4,5dimethylthiazolyl-2)-2,5-diphenyltetrazolium bromide (MTT) assay (9) upon transfection of Hep3B and HepG2 with miR-371 mimics or inhibitor. Our results showed that the expression of miR-371 was greatly increased in Hep3B cells transfected with miR-371 mimics (Fig. 2A), and markedly decreased in Hep3B cells transfected with miR-371 inhibitor (Fig. 2B), compared to negative control $(\mathrm{NC})$ as determined by qPCR. We found that the transfection of miR-371 mimics promoted the proliferation of Hep3B (Fig. 2C) and HepG2 (Fig. 2D), whereas the transfection of miR-371 inhibitor repressed the proliferation of Hep3B (Fig. 2E) and HepG2 (Fig. 2F). We also used soft agar colony formation assay $(10,11)$ to assess the impact of miR-371 overexpression on human hepatoma cells. In addition, we observed that transfection of miR-371 mimics promoted the proliferation of Hep3B (Fig. 2G and Fig. 2H) and HepG2 (Fig. 2I and Fig. 2J), whereas the transfection of miR-371 inhibitor repressed the proliferation of Hep3B cells (Fig. 2K).

\section{MiR-371 promotes migration and invasion of HCC cells}

Since a positive correlation was observed in the HCC dataset between miR-371 expression and metastasis, we studied the

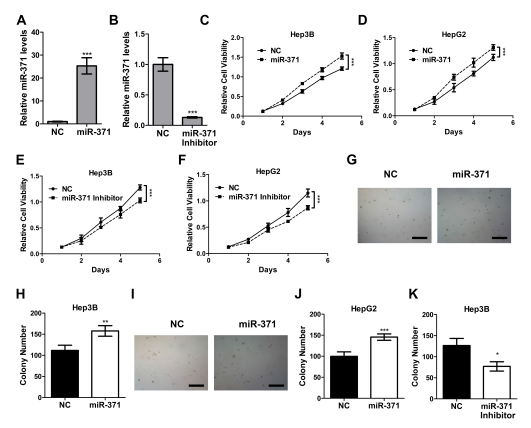

Fig. 2. miR-371 promotes HCC cells proliferation. (A) The expression of miR-371 in Hep3B cell transfected with miR-371 or $\mathrm{NC}$ as determined by qPCR. (B) The expression of miR-371 in Hep3B cell transfected with miR-371 inhibitor or NC as determined by qPCR. (C) The proliferation of Hep3B cells transfected with miR-371 mimics as determined by MTT assay. (D) The proliferation of HepG2 cells transfected with miR-371 mimics as determined by MTT assay. (E) The proliferation of Hep3B cells transfected with miR-371 inhibitor as determined by MTT assay. (F) The proliferation of HepG2 cells transfected with miR-371 inhibitor as determined by MTT assay. (G) The proliferation of Hep3B cells transfected with miR-371 mimics as determined by soft agar colony formation assay. Scale bar: 500 $\mu \mathrm{m}$. (H) The statistical results of soft agar colony formation assay. (I) The proliferation of HepG2 cells transfected with miR-371 mimics as determined by soft agar colony formation assay. Scale bar: $500 \mu \mathrm{m}$. (J) The statistical results of soft agar colony formation assay. (K) The proliferation of Hep3B cells transfected with miR-371 inhibitor as determined by soft agar colony formation assay. Data are shown as mean \pm S.D. $* P<0.05$; $* * \mathrm{P}<0.01 ; * * * \mathrm{P}<0.001 ; \mathrm{ns}$, not significant (Student's t-test).

impact of miR-371 on HCC metastasis. First, we conducted a Transwell invasive assay (12) to evaluate the effects of miR-371 on HCC cell migration and invasion. We found that the expression of miRNA-371 was higher in cells on the lower side of the insert than those in the upper chamber of Transwell filters in Hep3B cells (Fig. 3A) and HepG2 cells (Fig. 3B). In addition, the transfection of miR-371 promoted the migration and invasion of Hep3B cells (Fig. 3C and Fig. 3D) and HepG2 cells (Fig. 3E and Fig. 3F). These results suggest that miR-371 promoted HCC metastasis.

\section{PTEN is a direct target of miR-371}

In order to identify the targets of miR-371, we first used TargetScan (http://www.targetscan.org/vert_71/) to predict the potential targets of miR-371 (13), which identified about 400 conserved potential target genes of miR-371. We then selected seven genes that had been previously implicated in miRNA-mediated tumorigenesis for further study. We explored the impact of miR-371 on the expression of these seven genes in HCC cells using qPCR. We found that three genes, including occludin (OCLN), lymphoid enhancer binding factor 1 (LEF1), and phosphatase and tensin homolog (PTEN), were down-regulated by miR-317 in both Hep3B cells and HepG2 


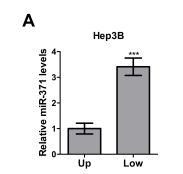

D
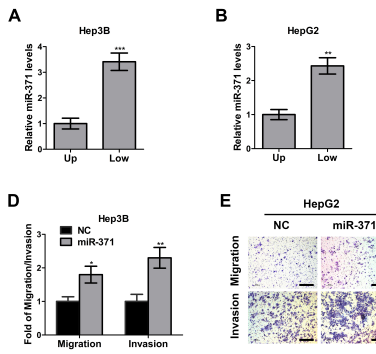

E $\quad$ HepG2
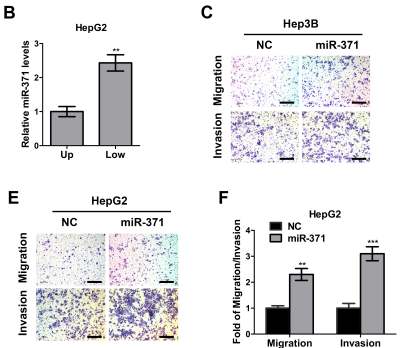

Fig. 3. miR-371 promotes migration and invasion of HCC cells. (A) The expression of miR-371 in invasive Hep3B cells versus non-invasive Hep3B cells. (B) The expression of miR-371 in invasive HepG2 cells versus non-invasive HepG2 cells. (C) Transwell migration and invasion assay of Hep3B cells transfected with miR-371 mimics. Scale bar: 200 um. (D) The statistical results of Transwell migration and invasion assay. (E) Transwell migration and invasion assay of HepG2 cells transfected with miR-371 mimics. Scale bar: $200 \mu \mathrm{m}$. (F) The statistical results of Transwell migration and invasion assay. Data are shown as mean \pm S.D. ${ }^{*} \mathrm{P}<0.05 ; * * \mathrm{P}<0.01 ; * * * \mathrm{P}<0.001 ;$ ns, not significant (Student's t-test).

cells (Fig. 4A). We therefore used luciferase reporter assay to further examine the activities driven by the 3'-untranslated regions ( $3^{\prime}$ UTRs) of potential miR-371 targets in Hep3B cells transfected with miR-371 mimics or negative control (NC), in order to test whether the above three genes were directly targeted by miR-317. Indeed, we found that miR-371 only repressed the reporter activities of 3'UTRs of PTEN, implicating PTEN as a direct target of miR-371 (Fig. 4B). We then predicted the potential binding site of miR-371 on PTEN 3'UTR using TargetScan. Luciferase reporter assay showed that the repression of the reporter activities of PTEN 3'UTRs was eliminated when the predicted binding site was mutated, indicated that PTEN may be a bona fide direct target of miR-371 (Fig. 4C and Fig. 4D). Furthermore, we found that miR-371 repressed the protein expression level of PTEN in Hep3B cells and HepG2 cells (Fig. 4E).

\section{Overexpression of PTEN inhibits HCC cell proliferation, migration and invasion}

We next studied the role of PTEN in HCC. We transfected Hep3B cells with PTEN overexpression plasmid, which significantly increased the mRNA level of PTEN (Fig. S1A). We found that PTEN overexpression in Hep3B cells markedly repressed the proliferation, as evidenced by MTT assay (Fig. S1B) or soft agar colony formation assay (Fig. S1C). We also found that PTEN overexpression in Hep3B cells repressed cell migration and invasion (Fig. S1D), whereas knockdown of PTEN by siRNA promoted (Fig. S1E) cell migration and invasion, as shown by Transwell migration and invasion assays. These results showed that PTEN repressed proliferation, migration, and invasion, and thus functionally recapitulated the role of miR-371 in HCC cells.

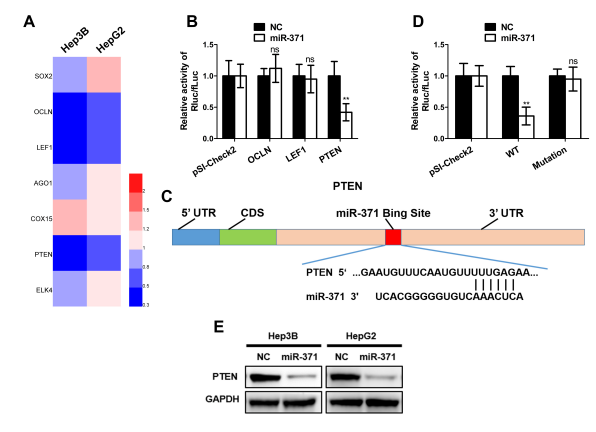

Fig. 4. PTEN is a direct target of miR-371. (A) Heat map representing the $m R N A$ expression profile of a panel of cancer-related genes regulated by miR-371 in Hep3B and HepG2 cell lines as determined using real-time PCR. (B) Luciferase reporter activities driven by the 3'UTRs of potential miR-371 targets were examined in Hep3B cells transfected with miR-371 mimics or negative control (NC). (C) Schematic diagram of miR-371 binding site on the 3'UTR of PTEN mRNA. (D) Luciferase reporter activities driven by wild-type or mutant PTEN 3'UTRs (TTTGAG > CAGCTA) were examined in Hep3B cells transfected with miR-371 mimics or negative control (NC). (E) Immunoblot analysis of PTEN protein level in Hep3B and HepG2 cells transfected with miR-371 mimics. Data are shown as mean \pm S.D. ${ }^{* P}<0.05 ;{ }^{* * P}<0.01 ;{ }^{* * * P}<0.001 ; \mathrm{ns}$, not significant (D: one-way ANOVA; others: Student's t-test).

\section{DISCUSSION}

MiRNAs negatively regulate gene expression, and can act as either tumor suppressors or oncogenes (14). The MiRNAs implicated in cancer have typically first been found to be differentially expressed in cancers, such as the miR-199a/214 cluster. In this work, we found that miR-371 played an important role in HCC, which extended the types of cancers in which miR-371 was implicated. Previous work has implicated miR-371 in pancreatic cancer cells, malignant germ cell tumors, and testicular germ cell tumors. Thus, miR-371 may serve some general function in cancer. Moreover, in other types of cancerous tissue such as esophageal squamous cell carcinoma, miR-371 is also elevated, and the cellular function is similar. For example, miR-317 also facilitates cell proliferation in pancreatic cancer. This suggests that miR-371 may have some conserved and important function in various types of tissues, which would mean that miR-371 is likely involved in more types of cancers.

By contrast, the miRNA target genes are not restricted to a particular miRNA. For example, in the current study, we identified PTEN as a direct target of miR-371. In fact, PTEN has already been reported to be a direct target of several other miRNAs. For instance, the expression of miR-21 promotes cell growth, metastasis, radio-resistance, or chemo-resistance in non-small cell lung cancer by targeting PTEN (15). In addition, MiR-221 and miR-222 promote gastric carcinoma cell proliferation and radio-resistance by targeting PTEN (16). Further, MiR-214 regulates the proliferation, migration, and 
invasion of gastric cancer cell by targeting PTEN (17). Not only does PTEN serve as a target for many miRNAs, but miRNAs can also have multiple targets, since miRNA pairing with mRNA is essentially only based on seven nucleotides within miRNA (miRNA seed). A single miRNA may target an array of different mRNAs; for example, miR-301 mediates proliferation and invasion in human breast cancer with FOXF2, BBC3, PTEN, and COL2A1 as its verified candidate targets (18). In this way, miRNAs form a layer of an interlaced network of regulation on their target genes. The target genes thus receive convergent signaling from, and are tightly regulated by, various miRNAs, which are themselves produced by RNA biogenesis. The tight control reflects the importance and delicate nature of this signaling. MiRNAs are highly conserved, and it is possible that the regulatory network evolves to accommodate the complicated coordination of intracellular signaling, which is most important in development and physiology.

The interconnected signaling regulations make functional study difficult, and network analysis is required for a comprehensive understanding of the functions in each node. The multi-target feature of miRNA also holds the potential that one particular node could influence a wide range of pathways that may orchestrate complex physiological processes in complex diseases such as cancer (3). Indeed, some enterprises initiated exploration to harness the potential power of miRNA in therapeutics (19), which requires manipulating an array of miRNAs for focused and/or coordinated targeting.

In the current study, we found PTEN to be a direct target of miR-371. However, the question is whether PTEN is the sole target of miR-317 in this setting, Although in the current assay, the phenotype observed by miR-371 are all in line with and can be explained by PTEN, we still could not exclude the other possibilities. More fine-grained analysis should clarify the problem and inform how the miR-371 might regulate certain aspects of cellular processes.

Analysis of the GSE6857 dataset has revealed that miR-371 shows higher expression in samples with metastasis and poor prognosis. Unlike primary tumors, metastatic cancers are hardly curable because they are often systemic and cannot be operated upon, therefore accounting for up to $90 \%$ of cancer-associated mortality (20).

In our in vitro study with HCC cell lines, we observed higher miR-371 expression in invasive cells than in noninvasive cells. In addition, the transfection of miR-371 turned the cells more invasive. MiR-371 and cell metastasis are inter-correlated, but a causative relationship could not be established. PTEN, the target of miR-371, also increases cell invasion, but still cannot explain why invasive cells have higher miR-371 expression. The non-straightforward relationship may reflect the complicated relationship between the miRNA and phenotype, because miRNAs mostly achieve their functions indirectly by acting on protein coding genes. We studied the function of miR-371 in vitro in HCC cell lines, and whether miR-371 works in normal liver cells or in vivo in the same way remains unknown. Further in vivo studies are needed to provide a more comprehensive understanding of the distribution and function of miR-371 in the liver or in other systems of animals or humans. Moreover, understanding the interactions between miR-371 and other HCC-related miRNAs should be informative for revealing the regulation by miRNAs.

\section{CONCLUSION}

MiR-371 is positively correlated with HCC metastasis and poor prognosis in HCC patients. MiR-371 promotes the proliferation, migration, and invasion of HCC cells. PTEN is a direct target of miR-371, and the overexpression of PTEN inhibits HCC cell proliferation, migration, and invasion.

\section{MATERIALS AND METHODS}

\section{Cell culture of Hep3B and HepG2}

We used two human hepatoma cell lines (Hep3B and HepG2) obtained from the Type Culture Collection of the Chinese Academy of Sciences. We cultured the cell lines in Dulbecco's modified Eagle's medium (DMEM; Hyclone, Marlborough, MA, USA) with supplements of $10 \%$ fetal bovine serum (FBS; Transgene, Beijing, China), $100 \mathrm{IU} / \mathrm{mL}$ penicillin, and $100 \mathrm{IU} / \mathrm{mL}$ streptomycin (Hyclone) in an incubator with $5 \% \mathrm{CO}_{2}$ at $37^{\circ} \mathrm{C}$.

\section{Cell transfection}

MiR-371/NC: we constructed a pcDNA3-miR-371 plasmid by inserting into pcDNA3 a DNA fragment containing miR-371 which was amplified from human genomic DNA by PCR with the following primers: forward 5'-GCCTGCTCGAGAAAGGG TCGTTAAATTCGTGC-3' and reverse 5'-GCACGAAGCTTGA GCAGCTCCATCTGCAAGAG-3'. We used a synthesized antisense 20-O-methyl-modified oligonucleotide (5'-AGUGCCCC CACAGUUUGAGU-3') as miR-371 inhibitor. We used a scrambled sequence (5'-CAGUACUUUUGUGUAGUACAA-3') as negative control (NC).

PTEN-OE/NC: we constructed a PTEN over-expression plasmid by cloning into the pcDNA3 expression vector (Invitrogen, Carlsbad, CA, USA) with Lipofectamine ${ }^{\circledR} 2000$ the full-length PTEN CDNA, which was PCR amplified with the primer pair of forward: 5'-ATAGCGGCCGCCATGACAGCCAT CATCAAAG-3' and reverse: 5'-GTACTCGAGTTCCAATGAC TACACCATAAA-3' from a CDNA clone template derived from total RNA extracted from Hep3B and with EcoRI and Xhol. pcDNA3 empty vector was utilized as control. Hep3B cells were transfected with pcDNA3-PTEN or control pcDNA3 vector. All insertions were verified by DNA sequencing.

We synthesized PTEN siRNA duplexes (sense: GGCGCUAU GUGUAUUAUUAdTdT; antisense: UAAUAAUACACAUAGC GCCdTdT) and non-specific sequences (UUCUCCGAACGU GUCACGUdTdT) as siRNA negative control (NC).

We plated cells on 6-well or 24-well plates and transfected the cells using TurboFect Transfection Reagent (Thermo, NY, 
USA) (12) before harvesting them for RNA isolation, and used whole cell extracts for Western blot.

\section{MTT proliferation assay}

We used MTT cell viability assay to estimate the effect of miR-371/miR-371 inhibitor on the viabilities of Hep3B and HepG2, as well as the effect of PTEN on the viability of Hep3B. In order to derive viability/proliferation curves of the cells transfected with miR-371 or miR-371 inhibitor, or PTEN overexpression or knockdown, we seeded cells into a 96-well culture plate at about 500 cells/well, and allowed them to grow in DMEM with a $10 \%$ FBS supplement. We then used MTT reagent (Sigma, MO, USA) in $5 \mathrm{mg} / \mathrm{ml}$ PBS to measure the viabilities of the cells. We replaced the culture medium to fresh DMEM with 10\% FBS and diluted MTT $(1: 10,10 \%$ MTT), then incubated it for 3 or $5 \mathrm{~h}$ at $37^{\circ} \mathrm{C}$. We then removed the incubation medium and dissolved formazan crystals in 200 $\mu \mathrm{l}$ DMSO solution. We measured the light absorbance at 570 nm using a ELx800 absorbance microplate reader (BioTek Instruments, VT, USA) to quantify MTT reduction. We repeated each test at least four times and calculated the amount of MTT dye conversion relative to sham-treated control cells as the measure of cell viability (21).

\section{Soft agar colony formation assay}

We used 35-mm petri dishes to pour a 1.5-ml layer of DMEM with $0.5 \%$ agar (wt/vol) and 10\% FBS. We treated HepG2 and Hep3B cells for 24 hours with control or miR-371 at $100 \mathrm{nM}$ with Oligofectamine reagent (Invitrogen, USA), then resuspended them in DMEM with $0.35 \%$ agar (wt/vol) and 5\% FBS at 5000 cells $/ 1.5 \mathrm{ml}$ density. We poured the $1.5 \mathrm{ml}$ cell suspensions onto the base layer, allowed it to solidify, then incubated in $5 \% \mathrm{CO}_{2}$ at $37^{\circ} \mathrm{C}$ for 14 days (HepG2 cells) or 21 days (Hep3B cells). We stained the colonies with $0.005 \%$ crystal violet for one hour and counted the colonies containing more than 10 cells with an Olympus microscope (Olympus, Tokyo, Japan).

\section{Transwell assay}

We used Transwell assay to separate the migratory and non-migratory cells of Hep3B and HepG2. We used the Boyden chambers and placed cells $\left(1 \times 10^{5}\right.$ cells/well $)$ in the upper Transwell chambers ( $8 \mu \mathrm{m}$ pore size) with the lower Transwell chambers filled with $0.5 \mathrm{ml}$ DMEM containing 5\% FBS, then incubated the cells at $37^{\circ} \mathrm{C}$ in $5 \% \mathrm{CO}_{2}$ for $12 \mathrm{~h}$. The non-migratory cells (up) and cells on the bottom surface (bottom) were digested off, and to evaluate the miR-371 level in the migratory and non-migratory cells. We scraped the non-migratory cells with a cotton swab and then fixed them with $4 \%$ paraformaldehyde, stained them with $0.1 \%$ crystal violet (Beyotime, Shanghai, China), and counted the cells remaining on the bottom surface from five randomly selected fields at $200 \times$ magnification. We also used Boyden chambers for the cell invasion assay. We coated the upper surface of the filter with $20 \mu \mathrm{g}$ Matrigel $(0.3 \mathrm{mg} / \mathrm{ml}$; BD Biosciences, Franklin
Lakes, NJ, USA) and incubated cells at $37^{\circ} \mathrm{C}$ in $5 \% \mathrm{CO}_{2}$ for 24 hours, then counted the cells as described above.

\section{Westem blotting of PTEN}

We lysed the harvested cells (Hep3B or HepG2 transfected with miR-371 or NC) with radioimmunoprecipitation buffer containing phosphatase and protease inhibitor cocktails (PhosSTOP and cOmplete ULTRA Tablets, Roche, Penzberg, Upper Bavaria, Germany). The lysates were then boiled in sodium dodecyl sulfate (SDS) loading buffer, and separated by SDS-PAGE before being transferred to a nitrocellulose membrane for blocking in $5 \%$ nonfat milk containing phosphate-buffered saline (PBS)/Tween-20 at room temperature for $1 \mathrm{~h}$. We then incubated the membrane with primary antibodies specific for PTEN (\#9188, Cell Signaling Technology, Danvers, MA, USA 1:1,000), using glyceraldehyde 3-phosphate dehydrogenase (GAPDH) (ab9485, Abcam, Cambridge, MA, USA 1:2500) as internal control. The antigen-antibody complexes were detected using enhanced chemiluminescence (Pierce, IL, USA). We used secondary antibodies $(1: 10,000)$ from Bio-Rad (Hercules, CA, USA).

\section{Luciferase reporter assay}

Hep3B cells were co-transfected with $0.5 \mu \mathrm{g}$ of the reporter vector (psiCHECK-2, psiCHECK2-OCLN-3'UTR, psiCHECK2LEF1-3'UTR, psiCHECK2-PTEN-3'UTR with seed sequence TTTGAG, or psiCHECK-2-PTEN-Mut-3'UTR with seed sequence mutated to CAGCTA), in which the $3^{\prime} \cup T R$ sequences (PCR primers for amplification in Supplementary Table S1) were inserted into the plasmids using Xhol and Notl. First, $1 \mu \mathrm{g}$ of miR-371 expression plasmid or the control vector was used for each transfection. Then, 48 hours after transfection, we harvested the cells for the detection of luciferase activity with a dualluciferase reporter assay system (Promega, Fitchburg, WI, USA).

\section{Real Time PCR (RT-PCR)}

We isolated total RNA using Trizol reagent (Invitrogen, USA) following the manufacturer's instructions. In order to detect the expression of miR-371 in invasive (up) and non-invasive (bottom) Hep3B or HepG2 separated by the Transwell method, we used the Hairpin-it ${ }^{\mathrm{TM}}$ miRNAs qPCR Quantitation kit (GenePharma) for reverse transcription and quantitative PCR with U6 small nuclear RNA (snRNA) as an internal control for expression normalization. The thermal cycling conditions set for miR-371 and $\mathrm{U} 6$ snRNA were: $95^{\circ} \mathrm{C}$ for $3 \mathrm{~min}, 38$ cycles of $94^{\circ} \mathrm{C}$ for 12 $\mathrm{sec}$, and $63^{\circ} \mathrm{C}$ for $1 \mathrm{~min}$. We examined the expressions of SOX2, OCLN, LEF1, AGO1, COX15, PTEN, and ELK4 in Hep3B or HepG2 transfected with miR-371 or NC, and the expression of PTEN in Hep3B cells transfected with PTEN overexpression vector or empty vector by real-time PCR using the CFX96 Real-Time PCR Detection System (Bio-Rad, Hercules, CA, USA) and SYBR Premix Ex Taq II $(2 \times)$ (Tli RNaseH Plus, TaKaRa, Otsu, Japan). GAPDH mRNA levels were used for normalization. The relative expression of miR-371, snRNAs, or mRNAs was normalized using the comparative threshold cycle $\left(2^{-\Delta \mathrm{CT}}\right)$ 
method. The primer sequences used in this study were listed in the Supplementary Table S2.

\section{Bioinformatic analysis}

GSE6857 analysis: based on a normalized Gene Expression Omnibus (GEO) dataset of (HCC) specimens from radical resection GSE6857 $(22,23)$. The miRNA expression profile of HCC specimens was analyzed in order to explore the association of miR-371 with metastasis as well as the prognosis of HCC. MiR-371 target: using the TargetScan (v7.1; targetscan.org) (13), we identified a total of 403 transcripts with conserved sites as potential mRNA targets of miR-371. Among these potential targets, we chose seven conserved genes implicated in tumorigenesis for further study. The PTEN binding site was predicted by TargetScan.

\section{Statistical analysis}

Statistical analysis was carried out using Excel and SPSS. One-way ANOVA and Student's t-test were used for statistical comparison. The statistical significance level is set to 0.05 for P-value. All experiments were performed at least in triplicate.

\section{AUTHORS' CONTRIBUTIONS}

Hao Wang, Yi Zhao, Tingsong Chen, Guofang Liu, Nan He collected and assembled the data; Heping Hu performed the statistical analysis; Dawei Wang designed the study and wrote the manuscript. All authors read and approved the final submission.

\section{CONFLICTS OF INTEREST}

The authors have no conflicting interests.

\section{REFERENCES}

1. Llovet JM, Burroughs A and Bruix J (2003) Hepatocellular carcinoma. Lancet 362, 1907-1917

2. Bandiera S, Pfeffer S, Baumert TF and Zeisel MB (2015) miR-122-A key factor and therapeutic target in liver disease. J Hepatol 62, 448-457

3. Wiemer EAC (2007) The role of microRNAs in cancer: No small matter. Eur J Cancer 43, 1529-1544

4. Negrini M, Gramantieri L, Sabbioni S and Croce CM (2011) microRNA involvement in hepatocellular carcinoma. Anticancer Agents Med Chem 11, 500-521

5. Ghasemi M, Samaei NM, Mowla SJ, Shafiee M, Vasei M and Ghasemian N (2018) Upregulation of miR-371-373 cluster, a human embryonic stem cell specific microRNA cluster, in esophageal squamous cell carcinoma. J Cancer Res Ther 14, S132-S137

6. Murray MJ, Halsall DJ, Hook CE, Williams DM, Nicholson JC and Coleman N (2011) Identification of microRNAs From the miR-371 373 and miR-302 clusters as potential serum biomarkers of malignant germ cell tumors. Am J Clin Pathol 135, 119-125
7. Belge G, Dieckmann KP, Spiekermann M, Balks $T$ and Bullerdiek J (2012) Serum levels of microRNAs miR-371-3: a novel class of serum biomarkers for testicular germ cell tumors? Eur Urol 61, 1068-1069

8. Cairo S, Wang Y, de Reynies A et al (2010) Stem cell-like micro-RNA signature driven by Myc in aggressive liver cancer. Proc Natl Acad Sci U S A 107, 20471-20476

9. Stepanenko AA and Dmitrenko VV (2015) Pitfalls of the MTT assay: Direct and off-target effects of inhibitors can result in over/underestimation of cell viability. Gene 574, 193-203

10. Borowicz S, Van Scoyk M, Avasarala S et al (2014) The soft agar colony formation assay. J Vis Exp e51998

11. Horibata S, Vo TV, Subramanian V, Thompson PR and Coonrod SA (2015) Utilization of the soft agar colony formation assay to identify inhibitors of tumorigenicity in breast cancer cells. J Vis Exp e52727

12. Fu $X$, Wen $H$, Jing $L$ et al (2017) MicroRNA-155-5p promotes hepatocellular carcinoma progression by suppressing PTEN through the PI3K/Akt pathway. Cancer Sci 108, 620-631

13. Agarwal V, Bell GW, Nam JW and Bartel DP (2015) Predicting effective microRNA target sites in mammalian mRNAs. Elife 4, e05005

14. Winter J, Jung S, Keller S, Gregory RI and Diederichs S (2009) Many roads to maturity: microRNA biogenesis pathways and their regulation. Nat Cell Biol 11, 228-234

15. Zhang BG, Li JF, Yu BQ, Zhu ZG, Liu BY and Yan M (2012) microRNA-21 promotes tumor proliferation and invasion in gastric cancer by targeting PTEN. Oncol Rep 27, 1019-1026

16. Zhang C-Z, Han L, Zhang A-I et al (2010) MicroRNA-221 and microRNA-222 regulate gastric carcinoma cell proliferation and radioresistance by targeting PTEN. BMC Cancer 10, 367

17. Yang T-S, Yang $X-H$, Wang X-D, Wang Y-L, Zhou B and Song Z-S (2013) MiR-214 regulate gastric cancer cell proliferation, migration and invasion by targeting PTEN. Cancer Cell International 13, 68

18. Shi W, Gerster K, Alajez NM et al (2011) MicroRNA-301 Mediates Proliferation and Invasion in Human Breast Cancer. Cancer Res 71, 2926-2937

19. Mack GS (2007) MicroRNA gets down to business. Nat Biotechnol 25, 631-638

20. Valastyan S and Weinberg RA (2011) Tumor metastasis: molecular insights and evolving paradigms. Cell 147, 275-292

21. Fan SJ, Gao M, Meng QH et al (2005) Role of NF-kappa B signaling in hepatocyte growth factor scatter factormediated cell protection. Oncogene 24, 1749-1766

22. Budhu A, Roessler S, Zhao X et al (2013) Integrated metabolite and gene expression profiles identify lipid biomarkers associated with progression of hepatocellular carcinoma and patient outcomes. Gastroenterology 144, 1066-1075.e1061

23. Budhu A, Jia HL, Forgues $M$ et al (2008) Identification of metastasis-related microRNAs in hepatocellular carcinoma. Hepatology 47, 897-907 